\title{
On the Kinematic Signature of the Galactic Warp As Revealed By the LAMOST- TGAS Data
}

\author{
Y. Huang ${ }^{1,2,7}$ (1), R. Schönrich ${ }^{3}$, X.-W. Liu ${ }^{1}$, B.-Q. Chen ${ }^{1}$, H.-W. Zhang ${ }^{2,4}$, H.-B. Yuan ${ }^{5}$, M.-S. Xiang ${ }^{6,7}$, C. Wang ${ }^{2}$, and \\ Z.-J. $\operatorname{Tian}^{2,7}$ \\ ${ }^{1}$ South-Western Institute for Astronomy Research, Yunnan University, Kunming 650500, People's Republic of China; yanghuang@pku.edu.cn, x.liu@pku.edu.cn \\ ${ }^{2}$ Department of Astronomy, Peking University, Beijing 100871, People's Republic of China; zhanghw@ pku.edu.cn \\ ${ }^{3}$ Rudolf Peierls Centre for Theoretical Physics, 1 Keble Road, Oxford, OX1 3NP, UK; ralph.schoenrich@physics.ox.ac.uk \\ ${ }^{4}$ Kavli Institute for Astronomy and Astrophysics, Peking University, Beijing 100871, People's Republic of China \\ ${ }^{5}$ Department of Astronomy, Beijing Normal University, Beijing 100875, People's Republic of China \\ ${ }^{6}$ Key Laboratory of Optical Astronomy, National Astronomical Observatories, Chinese Academy of Sciences, Beijing 100012, People's Republic of China \\ Received 2018 February 15; revised 2018 July 4; accepted 2018 July 8; published 2018 September 7
}

\begin{abstract}
Using a sample of about 123,000 stars with accurate 3D velocity measurements from the LAMOST-TGAS data, we confirm the kinematic signature of the Galactic warp recently found by Schönrich \& Dehnen. The data reveal a clear trend of increasing mean vertical velocity $\bar{V}_{z}$ as a function of absolute vertical angular momentum $L_{z}$ and azimuthal velocity $V_{\phi}$ for guiding center radius $R_{g}$ between 6.0 and $10.5 \mathrm{kpc}$. The trend is consistent with a largescale Galactic warp. Similar to Schönrich \& Dehnen, we also find a wave-like pattern of $\overline{V_{z}}$ versus $L_{z}$ with an amplitude of $\sim 0.9 \mathrm{~km} \mathrm{~s}^{-1}$ on a scale of $\sim 2.0 \mathrm{kpc}$, which could arise from bending waves or a winding warp. Finally, we confirm a prominent, localized peak in $\bar{V}_{z}$ near $L_{z} \sim 2150 \mathrm{kpc} \mathrm{km} \mathrm{s}^{-1}$ (corresponding to $R_{g} \sim 9 \mathrm{kpc}$ and $V_{\phi} \sim 255 \mathrm{~km} \mathrm{~s}^{-1}$ ). The additional line-of-sight velocity information from LAMOST reveals that stars in this feature have a large, inward radial velocity of $V_{R} \sim-13.33 \pm 0.59 \mathrm{~km} \mathrm{~s}^{-1}$ and a small radial velocity dispersion of $\sigma_{R} \sim 25.27 \pm 0.89 \mathrm{~km} \mathrm{~s}^{-1}$, suggesting that a stellar stream gives rise to this feature.
\end{abstract}

Key words: Galaxy: kinematics and dynamics - Galaxy: structure - solar neighborhood - stars: distances - stars: kinematics and dynamics

\section{Introduction}

While the inner disks of most disk galaxies are remarkably flat, their outskirts are strongly warped. Consistent with expectations from simple approximations for vertical bending waves (Hunter \& Toomre 1969), the amplitude of these warps increases strongly with radius, reaching a level of a few inner disk scales in height. The warps are conspicuous in the neutral gas plane as revealed by $21 \mathrm{~cm} \mathrm{HI}$ observations (e.g., Sancisi 1976; Bosma 1981; Briggs 1990) and also seen in the stellar plane through optical and infrared observations (e.g., Sanchez-Saavedra et al. 1990; Reshetnikov \& Combes 1998; Saha et al. 2009). As a typical spiral galaxy, the Milky Way also displays significant warps in the outer disk as revealed by various tracers, including neutral gas (e.g., Burke 1957; Kerr 1957), molecular clouds (e.g., Grabelsky et al. 1987), stars (e.g., Drimmel \& Spergel 2001; López-Corredoira et al. 2002b), and even interstellar dust (e.g., Freudenreich et al. 1994; Drimmel \& Spergel 2001).

Theoretically, the warp in a spiral galaxy is generally considered to be a response of the galactic disk to a perturbation, which could come from the dark matter halo (assuming misaligned angular momenta; e.g., Ostriker \& Binney 1989; Debattista \& Sellwood 1999), the nearby satellite galaxies (such as the Sagittarius dwarf or the Large/Small Magellanic Clouds; e.g., Weinberg 1995; García-Ruiz et al. 2002; Bailin 2003), the cosmic infall (Jiang \& Binney 1999), or the intergalactic accretion flows (López-Corredoira et al. 2002a; Sánchez-Salcedo 2006). Currently, the exact origin of the Galactic warp is still under hot debate, although so many mechanisms have been proposed for its generation. The biggest

\footnotetext{
${ }^{7}$ LAMOST Fellow.
}

challenge for solving this problem is to explore the dynamic nature of the Galactic warp on the basis of its well-known structure.

At present, although several attempts (e.g., Miyamoto et al. 1988; Dehnen 1998; López-Corredoira et al. 2014; Poggio et al. 2017) have been made, particular subgroups or types of stars (i.e., OB stars or red clump stars), to study the kinematic behaviors of the Galactic warp, no definite conclusions have been reached due to the lack of sufficient proper motion data, either poor in accuracy for the ground-based or limited in number for space-borne Hipparcos (ESA 1997). Recently, the first Gaia data release (Gaia DR1; Gaia Collaboration et al. 2016b) from the Gaia survey (Gaia Collaboration et al. 2016a) provided accurate proper motion and parallax measurements of about two million stars by combining the data of Tycho and Gaia (TGAS; Lindegren et al. 2016). With this data set, Schönrich \& Dehnen et al. (2018; hereafter SD18) have estimated the vertical velocities $V_{z}$, azimuthal velocities $V_{\phi}$, and vertical angular momenta $L_{z}$ for stars in the Galactic center and anti-center directions. They find that the mean vertical velocity $\overline{V_{z}}$ shows a clear increasing trend with both $V_{\phi}$ and $L_{z}$, as expected from a long-lived Galactic warp (e.g., Drimmel et al. 2000; hereafter DSL00). Moreover, a previously unknown wave-like pattern of $\overline{V_{z}}$ versus $L_{z}$ (and versus $V_{\phi}$ and guiding center radius $R_{g}$ ) with an amplitude of $\sim 1 \mathrm{~km} \mathrm{~s}^{-1}$ on a scale of $\sim 2.5 \mathrm{kpc}$ is detected. They suggest that the feature could arise from a winding warp or bending waves. In addition, a stream-like feature near $R_{g} \sim 9 \mathrm{kpc}\left(L_{z} \sim 2150 \mathrm{kpc} \mathrm{km} \mathrm{s}^{-1}\right)$ is found to deviate significantly from this wave-like pattern.

In this paper, we have repeated the analysis of SD18 using full three-dimensional (3D) velocity measurements (tangential 


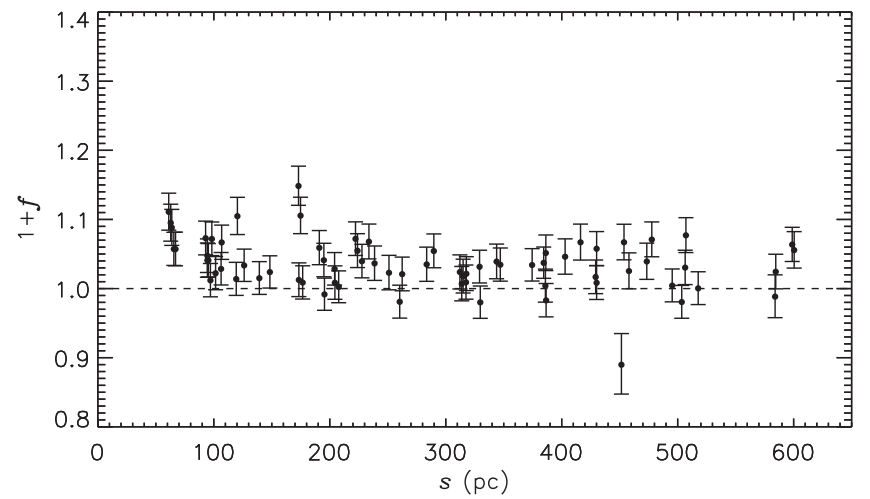

Figure 1. Distance estimator $1+f$ vs. distance $s$ for the the LAMOST-TGAS stars used in this work. The stars are binned in distance such that each bin contains 3000 stars, and the distance mask slides in steps of 1000 stars. For the definition of estimator $1+f$, please refer to SBA. In principle, $1+f$ should be unity if there are no systematic biases in the estimated distances.

plus line-of-sight velocities) by combining the LAMOST and TGAS data, rather than tangential velocities only as in SD18. The accurate $3 \mathrm{D}$ velocity measurements for a large number of stars available from the LAMOST-TGAS data provide a direct check of the results of SD18. The paper is organized as follows. In Section 2, we briefly describe the LAMOST-TGAS data set. The results are presented and discussed in Section 3. Finally, we summarize in Section 4.

\section{Data \\ 2.1. Coordinate Systems}

In this study, we use the standard Galactocentric cylindrical system $(R, \phi, z)$ with $R$, the projected Galactocentric distance, increasing radially outward; $\phi$ in the direction of Galactic rotation, and $z$ toward the North Galactic Pole. The three velocity components are, respectively, represented by $V_{R}, V_{\phi}$, and $V_{z}$. The guiding center is defined as $R_{g}=R V_{\phi} / V_{c}\left(R_{0}\right)$, where $R_{0}$ is the Galactocentric distance of the Sun, given the fact that the Galactic rotation curve in the solar neighborhood is nearly flat (e.g., McMillan 2011; Reid et al. 2014; Huang et al. 2016). For the solar motions and $R_{0}$, we use the same values adopted by $\mathrm{SD} 18$, i.e., $V_{\phi, \odot}=250 \mathrm{~km} \mathrm{~s}^{-1},\left(U_{\odot}, V_{\odot}\right.$, $\left.W_{\odot}\right)=(13.00,12.24,7.24) \mathrm{km} \mathrm{s}^{-1}$ and $R_{0}=8.27 \mathrm{kpc}$, determined by Schönrich et al. (2010) and Schönrich (2012), and consistent with results of Reid \& Brunthaler (2004), Reid et al. (2014), and Huang et al. (2015a, 2016), resulting in $V_{c}\left(R_{0}\right)$ is $238 \mathrm{~km} \mathrm{~s}^{-1}$.

\subsection{LAMOST and TGAS Data}

In this work, we use the data from two surveys: the LAMOST Galactic spectroscopic survey and the Gaia survey.

Full descriptions (including scientific motivations and target selections) of the LAMOST Galactic spectroscopic surveys are presented in Zhao et al. (2012), Deng et al. (2012), and Liu et al. (2014). The LAMOST Phase-I Surveys were initiated in the fall of 2012 (after one year of Pilot Surveys from 2011 September to 2012 June) and completed in the summer of 2017. The Phase-II Pilot Surveys kicked off last September, with the Phase-II Regular ones expected to begin in the coming September for another period of five years. At present, with the stellar parameter determination pipeline LSP3 (Xiang et al. 2015), line-of-sight velocities and basic atmospheric parameters (effective temperature $T_{\text {eff }}$, surface gravity $\log g$, and metallicity
$[\mathrm{Fe} / \mathrm{H}])$ have been derived from approximately 6.5 million qualified (i.e., of signal-to-noise ratios- $\mathrm{S} / \mathrm{Ns}$ greater than 10 ) stellar spectra of about 4.4 million stars, collected with LAMOST by 2016 June. This data set will soon be publicly available as a value-added catalog for LSS-GAC DR3.

The European Space Agency (ESA) Gaia satellite was launched in 2013 December. It will obtain highly accurate parallaxes and proper motions for nearly $1 \%$ ( one billion) of all stars in the Milky Way of a $G$ magnitude brighter than 20.7 (Gaia Collaboration et al. 2016a). With early survey data, the Gaia DR1 (Gaia Collaboration et al. 2016b) was released to the community on 2016 September 14. The astrometric TGAS catalog provides parallaxes and proper motions for over two million stars from the Tycho and Hipparcos catalogs (Lindegren et al. 2016).

We have cross-matched the LSS-GAC DR3 with the Gaia TGAS and found nearly 0.25 million common stars of LAMOST spectral S/Ns greater than 10. To ensure accurate $3 \mathrm{D}$ velocities, we have further applied the following cuts to the LAMOST-TGAS sample of common stars:

1. LAMOST spectral S/Ns greater than 30;

2. metallicity $[\mathrm{Fe} / \mathrm{H}] \geqslant-1.0 \mathrm{dex}$; and

3. relative parallax errors smaller than $20 \%$ in TGAS.

The first cut ensures that the precision of the LAMOST line-ofsight velocities is better than $5 \mathrm{~km} \mathrm{~s}^{-1}$ (Huang et al. 2018). Note that line-of-sight velocities yielded by LSP3 have been corrected for a systematic offset of $3.1 \mathrm{~km} \mathrm{~s}^{-1}$ (Xiang et al. 2015). The second cut is used to exclude potential contaminations from the halo stars. On top of the third cut, we have adopted the distance estimates of Schönrich \& Amuer (2017; hereafter SA17), who consider both the distance prior and the survey selection function prior to deriving the distances from the TGAS parallaxes. For the validation of the derived distances, we use the method of Schönrich et al. (2012; hereafter SBA) and find that the potential systematic uncertainties are smaller than a few percent (overestimated by $\sim 3.3 \%$, see Figure 1 ). The distance bias is small and the measurement may be affected by the residual uncertainties in the determination of LAMOST line-of-sight uncertainties. More importantly, the statistics are very stable versus distance, affirming the quality of the data set. Our data are less prone to distance bias than a mere proper motion sample as used in SD18, and a $3 \%$ distance offset should result in a general vertical velocity bias of less than $0.4 \mathrm{~km} \mathrm{~s}^{-1}$, as also tested in our analysis. Therefore, we can refrain from further corrections. With the third cut and the SA17 technique, precise and bias-free distances have been determined for 123,233 LAMOST-TGAS common stars after applying the first two cuts. With the above estimated distances, together with the line-of-sight velocities ${ }^{8}$ from the LSS-GAC DR3 and the proper motions from the TGAS, we further derive the 3D $(R, \phi, z)$ positions, and $3 \mathrm{D}\left(V_{R}, V_{\phi}, V_{z}\right)$ velocities, for these stars. Finally, the values of guiding center radius $R_{g}$ and vertical angular momentum $L_{z}$ are, respectively, calculated using $R_{g}=R V_{\phi} / V_{c}\left(R_{0}\right)$ and $L_{z}=R V_{\phi}$.

The spatial distribution of the final LAMOST-TGAS sample of common stars is presented in Figure 2. As the plot shows, the sample is restricted to within $\sim 0.6 \mathrm{kpc}$ of the Sun, but guiding center radii are probed in the range of $5<R_{g}<10 \mathrm{kpc}$. The short distance range of the sample also restricts the vertical extent and thus limits contamination of our signal by breathing

\footnotetext{
8 The LAMOST line-of-sight velocities are also tested bias free on the level of $2 \mathrm{~km} \mathrm{~s}^{-1}$ by the SBA method.
} 


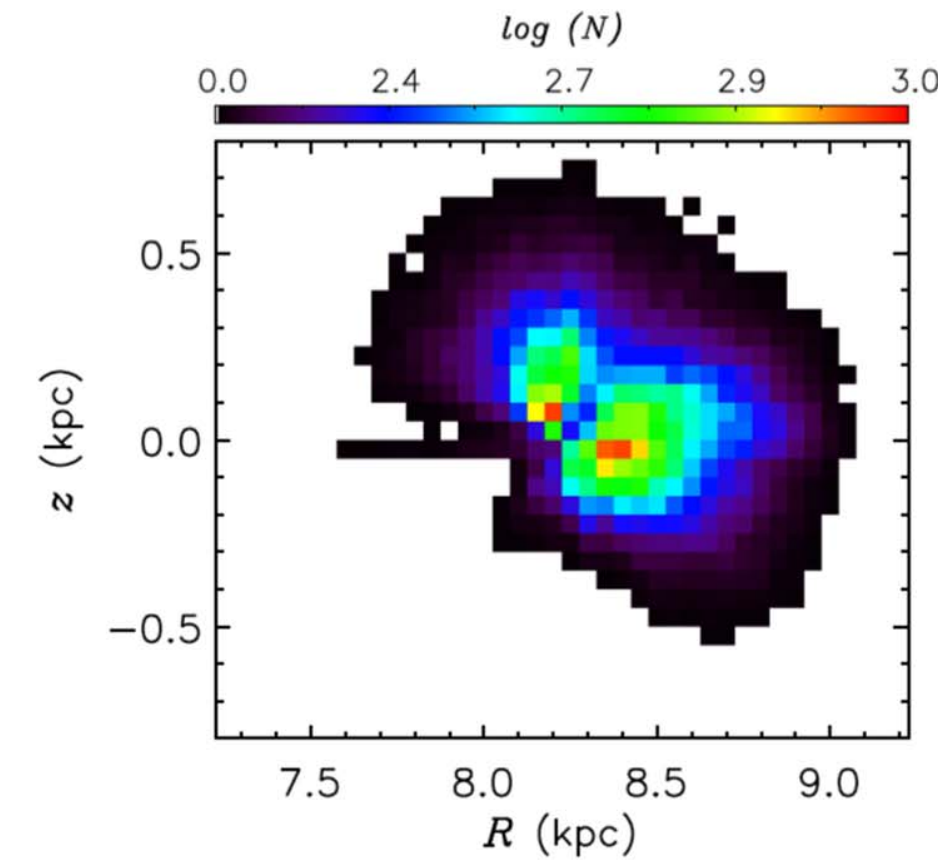

$$
\log (N)
$$

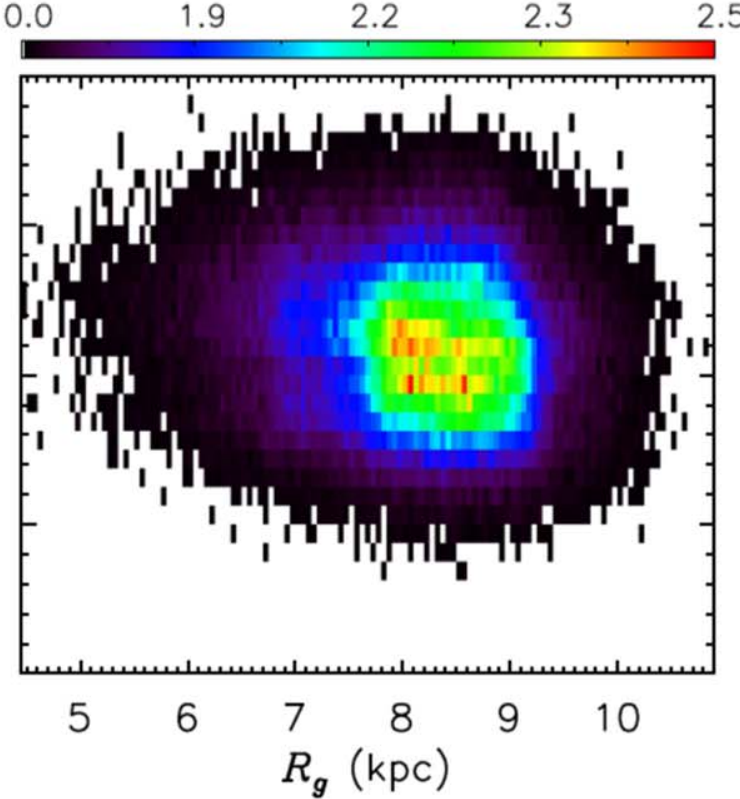

Figure 2. Spatial distribution of the final LAMOST-TGAS common stars in the $R-z$ (left) and $R_{g}-z$ (right) planes. The stellar number densities (with a bin size of $0.05 \mathrm{kpc}$ in each axis) are indicated by the top colorbars.

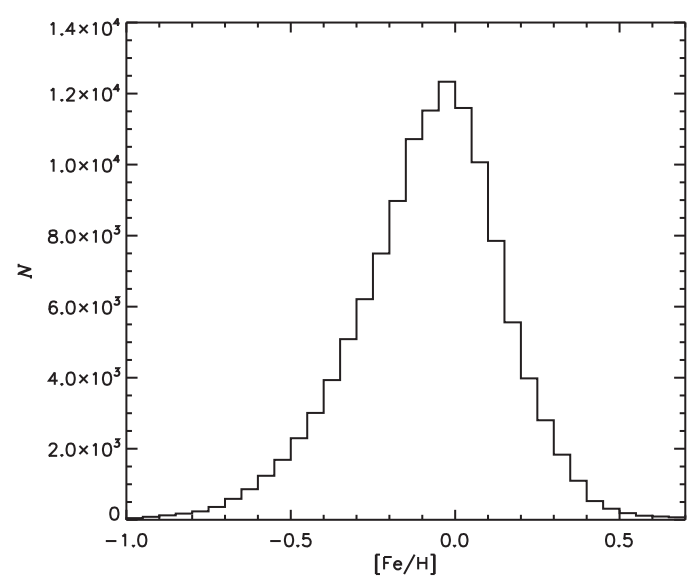

Figure 3. Metallicity distribution of the final selected 123,233 LAMOSTTGAS common stars.

modes. The metallicity distribution of the final selected sample is shown in Figure 3. The median of the distribution is around -0.06 dex, indicating a thin disk population nature of the sample. A plot of the sample in the $T_{\text {eff }}-M_{V}$ plane is shown in Figure 4. Here the effective temperatures are estimated from color $\left(V-K_{\mathrm{s}}\right)_{0}$, using the empirical metallicity-dependent calibration relations provided by Huang et al. (2015b). We have also adopted the values of the interstellar reddening of the individual stars derived with the "standard pair" technique (Yuan et al. 2013).

\section{Results and Discussion}

Systematic motions perpendicular to the Galactic plane will be induced if there is a long-lived warp in the stellar disk. Typically, the systematic vertical motions show a trend to increase with $R$ on a large scale (e.g., DSL00). As mentioned above, our sample covers a wide range of $R_{g}$ but not $R$.

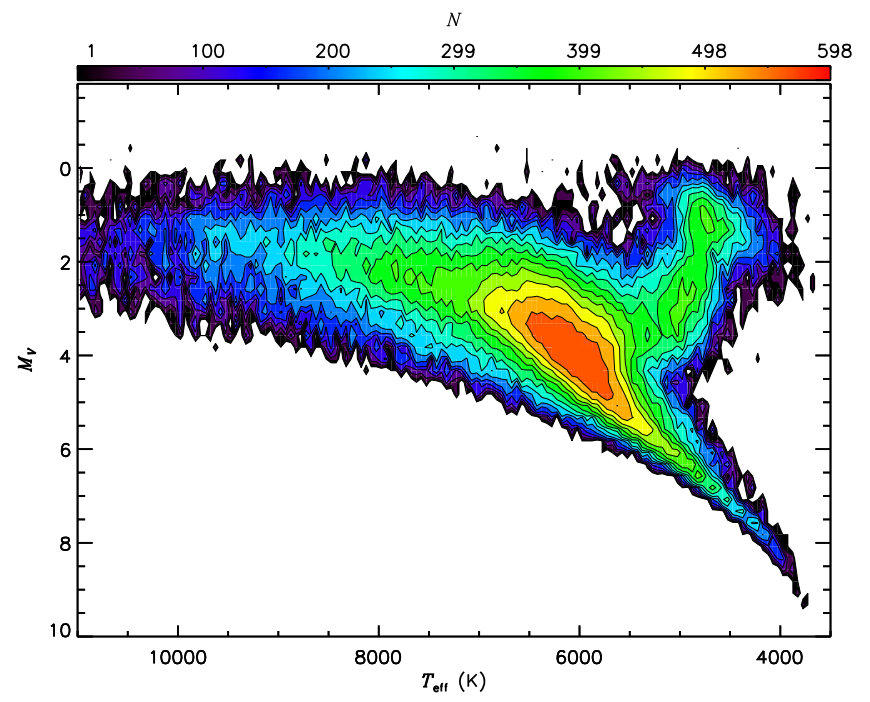

Figure 4. Pseudo-color $T_{\text {eff }}-M_{\mathrm{V}}$ diagram of the final selected 123,233 LAMOST-TGAS common stars. The stellar number density is indicated by the top colorbar.

Following SD18, we therefore study the mean vertical motions of our sample as a function of $L_{z}, R_{g}$, and $V_{\phi}$.

\subsection{The Kinematic Signature of the Galactic Warp}

As in SD18, we examine the mean values of vertical velocity $\bar{V}_{z}$ of our final LAMOST-TGAS sample in the individual bins of vertical angular momentum $L_{z}$. The bin size in $L_{z}$ is allowed to vary to ensure no less than 100 stars in each bin but set to be no smaller than $50 \mathrm{kpc} \mathrm{km} \mathrm{s}^{-1}$. The latter is set to match the typical uncertainties of $L_{z}$. The error bar of $\bar{V}_{z}$ for a given bin is given by $\sigma_{V_{z}} / \sqrt{N}$, where $\sigma_{V_{z}}$ is the measured vertical velocity dispersion and $N$ is the total number of stars in that 

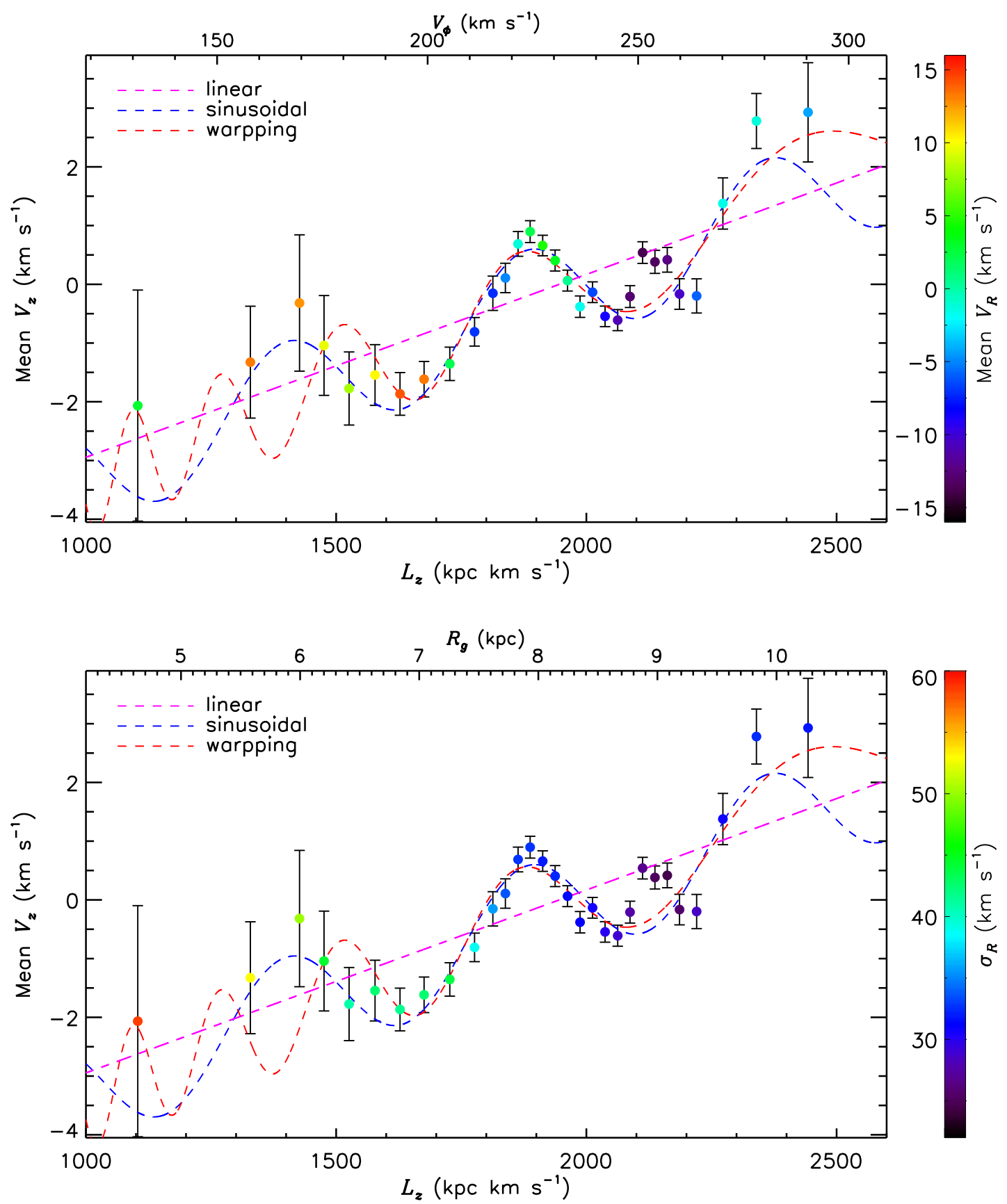

Figure 5. Mean $V_{Z}$ velocity vs. $L_{z}$, azimutal velocity $V_{\phi}$, or guiding center radius $R_{\mathrm{g}}$ for the final LAMOST-TGAS sample. The magenta, blue, and red dash lines represent a linear fit from Equation (1), a simple sinusoidal fit from Equation (2), and a wrapping fit from Equation (3), respectively, and the best-fit parameters are presented in Table 1 . The colors of the data points show the values of mean radial velocity $V_{R}$ (top panel) and radial velocity dispersion $\sigma_{R}$ (bottom panel) of each angular momentum bin, respectively. The bin size is set to be no smaller than $50 \mathrm{kpc} \mathrm{km} \mathrm{s}^{-1}$ but allowed to be larger to ensure no less than $100 \mathrm{stars}$ in each bin.

Table 1

Best-fit Parameters of Three Models, Described by Equations (1)-(3) Fit to the LAMOST-TGAS Data As Shown in Figure 2

\begin{tabular}{lccrr}
\hline \hline Fit & $\begin{array}{c}a \\
\left(\times 10^{3} \mathrm{kpc}\right)\end{array}$ & $\begin{array}{c}b \\
\left(\mathrm{~km} \mathrm{~s}^{-1}\right)\end{array}$ & $\begin{array}{c}c \\
\left(\mathrm{kp} \mathrm{km} \mathrm{s}^{-1}\right)\end{array}$ & $\begin{array}{c}A \\
\left(\mathrm{~km} \mathrm{~s}^{-1}\right)\end{array}$ \\
\hline linear & $3.11 \pm 0.70$ & $-1.078 \pm 0.273$ & $\ldots$ & $\ldots$ \\
sinusoidal & $3.24 \pm 0.38$ & $-1.277 \pm 0.149$ & $481 \pm 20$ & $-2.05 \pm 0.22$ \\
wrapping & $3.47 \pm 0.44$ & $-1.283 \pm 0.174$ & $7951 \pm 341$ & $-0.12 \pm 1.13$ \\
\hline
\end{tabular}

bin. The results are shown in Figure 5, with $V_{\phi}$ (assuming a mean radius $\bar{R}$ of $\sim 8.41 \mathrm{kpc}$ of the sample) and $R_{g}$ (assuming a flat rotation curve with a local circular speed of $238 \mathrm{~km} \mathrm{~s}^{-1}$ ) also labeled.
The overall mean vertical velocity $\bar{V}_{z}$ shows a significant increase and a wave-like pattern over $L_{z}, V_{\phi}$, and $R_{g}$, in excellent agreement with the result derived by SD18 using the TGAS data only and also the expectation of a large-scale warp 

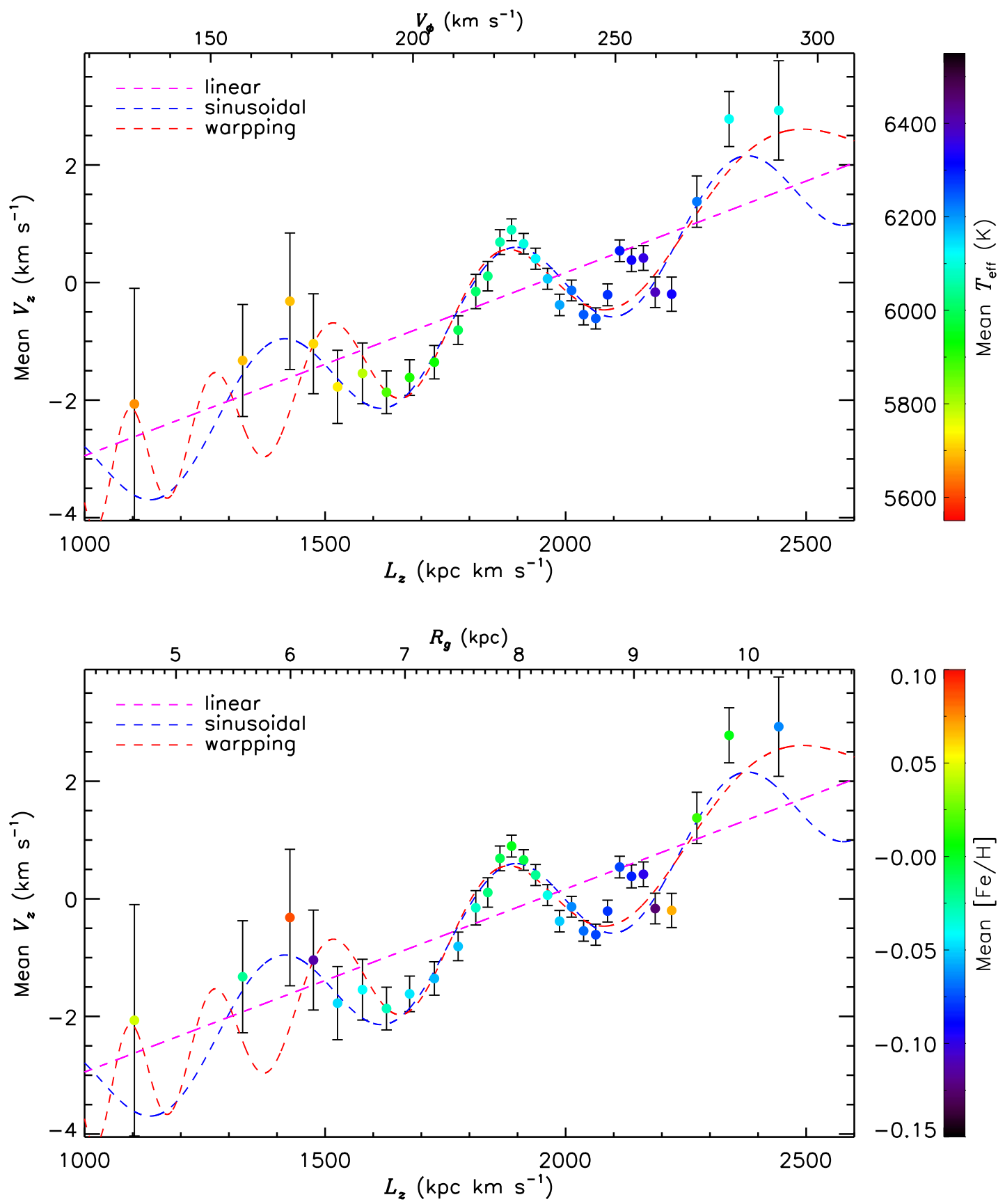

Figure 6. Similar to Figure 2 but with colors coded by mean $T_{\text {eff }}$ (top panel) and by mean $[\mathrm{Fe} / \mathrm{H}]$ (bottom panel).

signal in the stellar disk (e.g., DSL00). The trend in $\bar{V}_{z}$ versus $R_{g}$ shows a large-scale warp signal for $R_{g}$ ranging from about 6.0 to $10.5 \mathrm{kpc}$. This places a strong constraint on the start position of the Galactic warp. The onset radius of the galactic warp is highly debated, with estimates ranging from within, at, or outside the solar annulus (Derriere \& Robin 2001; Drimmel $\&$ Spergel 2001). Our results place a strong constraint on the onset point, significantly within the solar annulus, and thus is consistent with what was found by Drimmel \& Spergel (2001) with near-infrared photometry.

The feature at $L_{z} \sim 2150 \mathrm{kpc} \mathrm{km} \mathrm{s}^{-1}$ (corresponding to $R_{g} \sim 9 \mathrm{kpc}$ and $V_{\phi} \sim 255 \mathrm{~km} \mathrm{~s}^{-1}$ ) is suspiciously narrow and likely a stream, so we censor the region from the following fits. We will return to this question in Section 3.3.

\subsection{Fits with Simple Models}

In this subsection, we attempt to fit our new data with three simple models first proposed by SD18.

If one assumes a simple, perfectly static, nonwrapped warp model (e.g., DSL00, Poggio et al. 2017), the correlation between $\overline{V_{z}}$ and $L_{z}$ can be locally approximated by a linear fit:

$$
\overline{V_{z}}=b+a L_{z}^{\prime},
$$

where $L_{z}^{\prime}=L_{z}-1600 \mathrm{kpc} \mathrm{km} \mathrm{s}{ }^{-1}$.

The above pure linear relation only described the general trend of increase of $\bar{V}_{z}$ with $-L_{z}$ but not the wave-like pattern. For the latter, two simple yet physically motivated models are considered: (1) the disk may oscillate vertically as radially propagating waves (namely bending waves; Hunter \& Toomre 1969); and (2) the 
Galactic warp is excited by perturbations of dwarf satellites (e.g., the Sagittarius or the Magellanic Clouds) falling into the Galactic halo (namely a wrapping/winding warp; Weinberg 1995; Weinberg \& Blitz 2006). For the former, one has the following simple relation,

$$
\overline{V_{z}}=b+a L_{z}^{\prime}+A \sin \left(2 \pi L_{z}^{\prime} / c+d\right),
$$

where $A$ is the amplitude of the wave-like pattern, $c$ is the period, and $d$ is the phase. For the second model, the high stellar vertical oscillation frequencies compared to the Magellanic Clouds' orbital frequencies lead to the warp wrapping up, with the warp signal decreasing toward larger radii. Naively, we have the following relation for this model,

$$
\overline{V_{z}}=b+a L_{z}^{\prime}+A \sin \left(2 \pi c / L_{z}+d\right) .
$$

We fit the measured $\overline{V_{z}}-L_{z}$ variation with the three simple models described above. The fitting results are shown in Figure 5, with the best-fit parameters presented in Table 1. As the plot shows, the linear model describes the general trend of increase very well, but not the wave-like pattern. The other two models describe both perfectly. The amplitude of the wave-like pattern yielded by the latter two models is about $0.9 \mathrm{~km} \mathrm{~s}^{-1}$, similar to what was found by SD18. The radial wavelength of oscillation yielded by the simple sinusoidal model is about $2.0 \mathrm{kpc}$, again in excellent agreement with the result of SD18.

We note that one should not over-interpret the later two naive fits considering that the spatial coverage of the current sample is too limited to examine the underlying physics of both models. In addition, the range is too small to detect any changes in radial wavelength with radius, which is exemplified by both bracketing models working quite well. The future Gaia release, together with spectroscopic data (e.g., from LAMOST), will help solve this problem.

\subsection{A New Stellar Stream?}

Finally, we discuss the prominent localized peak at $L_{z} \sim 2150 \mathrm{kpc} \mathrm{km} \mathrm{s}^{-1}$ found here and earlier by SD18. As shown in Figure 5, the peak deviates significantly from all fits. To understand the nature of this peak, we show the mean radial velocity $\overline{V_{R}}$ and radial velocity dispersion $\sigma_{R}$ in the individual $L_{z}$ bins in Figure 5. As the plot shows, stars near the peak region have a significant inward mean radial motion (i.e., toward the Galactic center direction) of $-13.33 \pm 0.59 \mathrm{~km} \mathrm{~s}^{-1}$, while those outside the peak have motions that are close to zero. Stars near the peak region have a small radial velocity dispersion of only $25.27 \pm 0.89 \mathrm{~km} \mathrm{~s}^{-1}$, significantly smaller than the local radial velocity dispersion $\left(\sigma_{R 0} \sim 35 \mathrm{~km} \mathrm{~s}^{-1}\right.$; e.g., Huang et al. 2016). In addition, as Figure 6 shows, stars near this region also show a significant $T_{\text {eff }}$ peak $(\sim 6300 \mathrm{~K}$, about $100 \mathrm{~K}$ higher than the nearby bins) and have a $[\mathrm{Fe} / \mathrm{H}] \mathrm{dip}$ $(\sim-0.08,0.05-0.10$ dex lower than the nearby bins). These results strongly suggest that this localized peak arises from a young and cold stellar stream.

\section{Summary}

Using over 100,000 stars with accurate $3 \mathrm{D}$ velocity measurements selected from the LAMOST-TGAS data, we have repeated the work of SD18 (using tangential velocities from the TGAS only) to detect a kinematic signature of the Galactic warp.
With this data, a general trend of increase combined with a wave-like pattern of mean vertical velocity $\overline{V_{z}}$ versus absolute vertical angular momentum $L_{z}$ and azimuthal velocity $V_{\phi}$ is clearly detected for guiding center radius $R_{g}$ between $\sim 6.0$ and $10.5 \mathrm{kpc}$. The increase is expected from a large-scale Galactic warp. The wave-like pattern with an amplitude of $\sim 0.9 \mathrm{~km} \mathrm{~s}^{-1}$ on a scale of $\sim 2.0 \mathrm{kpc}$ may arise from bending waves or a winding/wrapping warp.

In addition to the above trend and wave-like pattern, a prominent localized peak is detected near $L_{z} \sim 2150 \mathrm{kpc} \mathrm{km} \mathrm{s}^{-1}$ (corresponding to $R_{g} \sim 9 \mathrm{kpc}$ and $V_{\phi} \sim 255 \mathrm{~km} \mathrm{~s}^{-1}$ ). It has a quite large inward radial velocity of $-13.33 \pm 0.59 \mathrm{~km} \mathrm{~s}^{-1}$ and a small radial velocity dispersion of $25.27 \pm 0.89 \mathrm{~km} \mathrm{~s}^{-1}$, suggesting a cold stellar stream nature of this feature.

In summary, this work and SD18 confirm an trend of increase associated with a wave-like pattern in $V_{z}$ over $L_{z}$. The result should provide vital constraints to future disk warp and (vertical) oscillation modeling.

The Guoshoujing Telescope (the Large Sky Area MultiObject Fiber Spectroscopic Telescope, LAMOST) is a National Major Scientific Project built by the Chinese Academy of Sciences. Funding for the project has been provided by the National Development and Reform Commission. LAMOST is operated and managed by the National Astronomical Observatories, Chinese Academy of Sciences. The LAMOST FELLOWSHIP is supported by the Special fund for Advanced Users, budgeted and administrated by the Center for Astronomical Mega-Science, Chinese Academy of Sciences (CAMS). R.S. is supported by a Royal Society University Research Fellowship.

This work has made use of data from the European Space Agency (ESA) mission Gaia (https://www.cosmos.esa.int/ gaia), processed by the Gaia Data Processing and Analysis Consortium (DPAC, https://www.cosmos.esa.int/web/gaia/ dpac/consortium).

This work is supported by the National Key Basic Research Program of China 2014CB845700, the China Postdoctoral Science Foundation 2016M600849, the National Natural Science Foundation of China U1531244, 11473001 and 11811530289. It is a pleasure to thank Zuhui Fan for valuable discussions.

\section{ORCID iDs}

\section{Y. Huang @ https://orcid.org/0000-0003-3250-2876}

\section{References}

Bailin, J. 2003, ApJL, 583, L79

Bosma, A. 1981, AJ, 86, 1825

Briggs, F. H. 1990, ApJ, 352, 15

Burke, B. F. 1957, AJ, 62, 90

Debattista, V. P., \& Sellwood, J. A. 1999, ApJL, 513, L107

Dehnen, W. 1998, AJ, 115, 2384

Deng, L.-C., Newberg, H. J., Liu, C., et al. 2012, RAA, 12, 735

Derriere, S., \& Robin, A. C. 2001, in ASP Conf. Ser. 232, The New Era of Wide Field Astronomy, ed. A. Clowes, A. Adamson, \& G. Bromage (San Francisco, CA: ASP), 229

Drimmel, R., Smart, R. L., \& Lattanzi, M. G. 2000, A\&A, 354, 67

Drimmel, R., \& Spergel, D. N. 2001, ApJ, 556, 181

ESA (ed.) 1997, ESA Special Publication 1200, The HIPPARCOS and TYCHO catalogues. Astrometric and photometric star catalogues derived from the ESA HIPPARCOS Space Astrometry Mission (Noordwijk: ESA) Freudenreich, H. T., Berriman, G. B., Dwek, E., et al. 1994, ApJL, 429, L69 Gaia Collaboration, Brown, A. G. A., Vallenari, A., et al. 2016a, A\&A, 595, A2

Gaia Collaboration, Prusti, T., de Bruijne, J. H. J., et al. 2016b, A\&A, 595, A1 
García-Ruiz, I., Kuijken, K., \& Dubinski, J. 2002, MNRAS, 337, 459

Grabelsky, D. A., Cohen, R. S., Bronfman, L., Thaddeus, P., \& May, J. 1987, ApJ, 315, 122

Huang, Y., Liu, X., Chen, B., et al. 2018, AJ, in press (arXiv:1806.06475)

Huang, Y., Liu, X.-W., Yuan, H.-B., et al. 2015a, MNRAS, 449, 162

Huang, Y., Liu, X.-W., Yuan, H.-B., et al. 2015b, MNRAS, 454, 2863

Huang, Y., Liu, X.-W., Yuan, H.-B., et al. 2016, MNRAS, 463, 2623

Hunter, C., \& Toomre, A. 1969, ApJ, 155, 747

Jiang, I.-G., \& Binney, J. 1999, MNRAS, 303, L7

Kerr, F. J. 1957, AJ, 62, 93

Lindegren, L., Lammers, U., Bastian, U., et al. 2016, A\&A, 595, A4

Liu, X.-W., et al. 2014, in Proc. IAU Symp. 298, Setting the Scene for Gaia and LAMOST, ed. S. Feltzing et al. (Cambridge: Cambridge Univ. Press), 310

López-Corredoira, M., Abedi, H., Garzón, F., \& Figueras, F. 2014, A\&A, 572, A101

López-Corredoira, M., Betancort-Rijo, J., \& Beckman, J. E. 2002a, A\&A, 386, 169

López-Corredoira, M., Cabrera-Lavers, A., Garzón, F., \& Hammersley, P. L. 2002b, A\&A, 394, 883

McMillan, P. J. 2011, MNRAS, 414, 2446

Miyamoto, M., Yoshizawa, M., \& Suzuki, S. 1988, A\&A, 194, 107
Ostriker, E. C., \& Binney, J. J. 1989, MNRAS, 237, 785

Poggio, E., Drimmel, R., Smart, R. L., Spagna, A., \& Lattanzi, M. G. 2017, A\&A, 601, A115

Reid, M. J., \& Brunthaler, A. 2004, ApJ, 616, 872

Reid, M. J., Menten, K. M., Brunthaler, A., et al. 2014, ApJ, 783, 130

Reshetnikov, V., \& Combes, F. 1998, A\&A, 337, 9

Saha, K., de Jong, R., \& Holwerda, B. 2009, MNRAS, 396, 409

Sanchez-Saavedra, M. L., Battaner, E., \& Florido, E. 1990, MNRAS, 246, 458

Sánchez-Salcedo, F. J. 2006, MNRAS, 365, 555

Sancisi, R. 1976, A\&A, 53, 159

Schönrich, R. 2012, MNRAS, 427, 274

Schönrich, R., \& Aumer, M. 2017, MNRAS, 472, 3979

Schönrich, R., Binney, J., \& Asplund, M. 2012, MNRAS, 420, 1281

Schönrich, R., Binney, J., \& Dehnen, W. 2010, MNRAS, 403, 1829

Schönrich, R., \& Dehnen, W. 2017, arXiv:1712.06616

Schönrich, R., \& Dehnen, W. 2018, MNRAS, 478, 3809

Weinberg, M. D. 1995, ApJL, 455, L31

Weinberg, M. D., \& Blitz, L. 2006, ApJL, 641, L33

Xiang, M. S., Liu, X. W., Yuan, H. B., et al. 2015, MNRAS, 448, 822

Yuan, H. B., Liu, X. W., \& Xiang, M. S. 2013, MNRAS, 430, 2188

Zhao, G., Zhao, Y.-H., Chu, Y.-Q., Jing, Y.-P., \& Deng, L.-C. 2012, RAA, 12,723 